\title{
Recent advances in cancer metabolism: a technological perspective
}

\author{
Yun Pyo Kang ${ }^{1}$, Nathan P. Ward ${ }^{1}$ and Gina M. DeNicola (id
}

\begin{abstract}
Cancer cells are highly dependent on metabolic pathways to sustain both their proliferation and adaption to harsh microenvironments. Thus, understanding the metabolic reprogramming that occurs in tumors can provide critical insights for the development of therapies targeting metabolism. In this review, we will discuss recent advancements in metabolomics and other multidisciplinary techniques that have led to the discovery of novel metabolic pathways and mechanisms in diverse cancer types.
\end{abstract}

\section{Introduction}

Cancer metabolism is profoundly different from normal cellular metabolism. Nearly 100 years ago, Otto Warburg described how cancer cells take up large amounts of glucose and metabolize it to lactate, even in the presence of oxygen, a phenotype not found in most normal tissues ${ }^{1}$. We now appreciate that metabolic reprogramming in cancers is complex, promotes the production of intermediates for the generation of new biomass, and is driven by mutations or epigenetic alterations in oncogenes, such as Ras, NRF2, and PIK3CA, or tumor suppressor genes, such as $\mathrm{p} 53, \mathrm{VHL}$, and $\mathrm{Rb}^{2}$. Tumor metabolism is also modified by cellular context, including interaction with other cells in the microenvironment, tumor hypoxia, and nutrient limitation ${ }^{2}$. It is also influenced by tissue-specific signaling, where the same oncogene can alter metabolism in one tissue but not another ${ }^{3}$. Metabolic reprogramming confers metabolic liabilities, which can be exploited for the therapeutic targeting for cancer therapy. Numerous therapies targeting metabolism are in use in the clinic, and these target multiple processes critical for tumor growth and survival, including nucleotide metabolism, amino acid metabolism, and carbon and fatty acid metabolism ${ }^{4}$.

\footnotetext{
Correspondence: Gina M. DeNicola (gina.denicola@moffitt.org)

'Department of Cancer Imaging and Metabolism, Moffitt Cancer Center and Research Institute, Tampa, FL 33612, USA

These authors contributed equally: Yun Pyo Kang, Nathan P. Ward.
}

In order to identify cancer-specific metabolic liabilities, an understanding of how metabolism is altered in both the tumor and microenvironment within specific tumor contexts is required. This is achieved through a combination of approaches that accurately measure metabolism in high-throughput, spatial and temporal manners. In this review we discuss recent technological advances in the study of cancer metabolism that have advanced our understanding of metabolic deregulation in cancer.

\section{Technologies for the study of metabolism}

Multiple analytical platforms that facilitate the detection of metabolites in cells and living organisms have been utilized to study cancer metabolism. In this section, we will discuss how these techniques have contributed to the study of cancer metabolism, and how they have led to advances in our understanding of metabolic reprograming and biological phenotypes.

\section{Chromatography coupled to mass spectrometry}

Since many of the intermediates associated with tumorpromoting metabolic pathways (e.g., glycolysis, tricarboxylic acid (TCA) cycle, and one-carbon metabolism) have hydrophilic chemical properties, analytical platforms that can measure these "water-soluble metabolites" are essential to understand cancer metabolism. In particular, chromatography (gas chromatography (GC) and liquid chromatography (LC)) coupled to mass spectrometry 
(MS)-based approaches are the most powerful techniques employed to determine the hydrophilic metabolite profiles of biological samples.

Sample preparation is an important consideration for metabolomics analysis. The extraction solvent must be chosen carefully based on its ability to extract the metabolites of interest. The metabolite extraction methods for multiple types of biological samples, such as cells and culture media, tissue, serum, and urine, have been optimized to extract a large number of metabolites, while concomitantly minimizing confounding alterations of their quantity and chemical structures prior to instrumental analysis ${ }^{5-12}$. These extraction considerations include the type of organic solvent, temperature, and acidity. For example, Rabinowitz et al. have optimized acetonitrile/methanol/water (40:40:20, v/v/v) containing $0.1 \mathrm{M}$ formic acid for cellular metabolite extraction, which enables global metabolite extraction while preventing degradation of nucleotide triphosphates such as $\mathrm{ATP}^{13}$.

Chromatography conditions are also an important consideration. For LC-based metabolite separation, both hydrophilic interaction liquid chromatography (HILIC) and ion-paring reagent-based reverse phase (RP) chromatography enable the separation of multiple hydrophilic metabolites, permitting qualitative and quantitative metabolite detection by $\mathrm{MS}^{14,15}$. During HILIC-based separation, the water-soluble metabolites interact with the hydrophilic stationary phase (Fig. 1) ${ }^{13}$. Alternatively, by applying an ion-pairing reagent consisting of both hydrophilic and hydrophobic residues in a single molecule, the hydrophilic metabolite can interact with the hydrophobic stationary phase of the RP column (Fig. 1) ${ }^{14}$. Metabolites are subsequently eluted from the column by applying a mobile phase gradient, during which the percentage of solvent (e.g., acetonitrile) increases in a timedependent manner, resulting in release of metabolites from the column based on their affinity (Fig. 1). Separation can be optimized by changing the mobile phase and gradient. For GC, in contrast, hydrophilic metabolite separation must be conducted after derivatization (e.g., trimethylsilylation) of hydrophilic residues to increase volatility ${ }^{16}$. GC utilizes a chemically inert gas as a mobile phase, and increasing the column oven temperature in a time-dependent manner facilitates metabolite elution. Thus, optimal metabolite separation is achieved through optimization of the temperature gradient.

The MS compartment can discriminate chemically similar metabolites that elute at the same time by their structural differences ${ }^{10,15}$. For LC-MS analysis, electrospray ionization (ESI) is used to ionize metabolites dissolved in the liquid mobile phase (Fig. 1). During ionization, the metabolites are charged by the high voltage of the probe, followed by their entry into the MS compartment. Furthermore, metabolites of interest can be distinguished from other chemically similar compounds by MS/MS during triple quadrupole-based targeted analysis or high-resolution MS (e.g., Q-TOF or Q-orbitrap) for non-targeted analysis (Fig. 1). For GC-MS analysis, electron impact (EI) is applied to ionize gas phase metabolites. In contrast to ESI, the EI method dissociates the intact metabolite ion, thereby generating multiple fragment ions. Since the intensity ratios of the fragment ions are very reproducible, the EI-MS pattern is used to achieve specificity during GC-MS-based metabolomics. Further, both GC-MS/MS and high-resolution MS (Q-Orbitrap) have been recently applied to conduct advanced metabolite analysis in a targeted or nontargeted way ${ }^{17,18}$. Finally, by comparing both the retention time from chromatographic separation and the MS specificity of the metabolite with an authentic standard, the metabolite identity can be determined.

Many metabolites such as amino acids can be detected by both LC-MS and GC-MS methods, while some metabolites can only be detected by one platform. For example, large molecular weight metabolites such as $\mathrm{CoA}$ and metabolites such as ATP or NADPH that are sensitive to high temperature can be only adequately detected by LC-MS ${ }^{19}$, but sugar alcohols and very short chain fatty acids are better separated and detected by GC-MS ${ }^{19}$. Thus, these two platforms have complementary roles to increase metabolite coverage when studying metabolism ${ }^{19}$.

The metabolite profiles acquired from LC-MS or GC-MS-based approaches have proved crucial for many recent discoveries related to cancer metabolism. For example, Mayers et al. conducted LC-MS-based plasma metabolite profiling and found that an elevation of branched-chain amino acids (BCAAs-isoleucine, leucine, and valine) occurs prior to the development of pancreatic ductal adenocarcinoma (PDAC) ${ }^{20}$. Using mouse models, they demonstrated these BCAAs were derived from whole-body protein degradation. Furthermore, global metabolite profiling was critical for the novel discovery of the oncometabolite 2-hydroxyglutarate (2-HG) in isocitrate dehydrogenase (IDH) mutant glioblastoma ${ }^{21}$. The global metabolite profile showed the significant elevation of a single metabolite peak in cells harboring a $\mathrm{R} 132 \mathrm{H}$ IDH1 mutation. The chemical formula acquired from high-resolution MS detection indicated the peak to be 2HG, and further targeted LC-MS-based analysis confirmed that the unknown metabolite was the $\mathrm{R}(-)$ stereoisomer of 2-HG (R(-)-2-HG). Importantly, subsequent studies have demonstrated the oncogenic role of $\mathrm{R}(-)-2-$ HG through the inhibition of alpha ketoglutaratedependent dioxygenases, including histone demethylases and 5-methlycytosine $(5 \mathrm{mC})$ hydroxylases ${ }^{22-25}$.

Unfortunately, there is no single analytical platform that provides suitable coverage for the whole metabolome, 


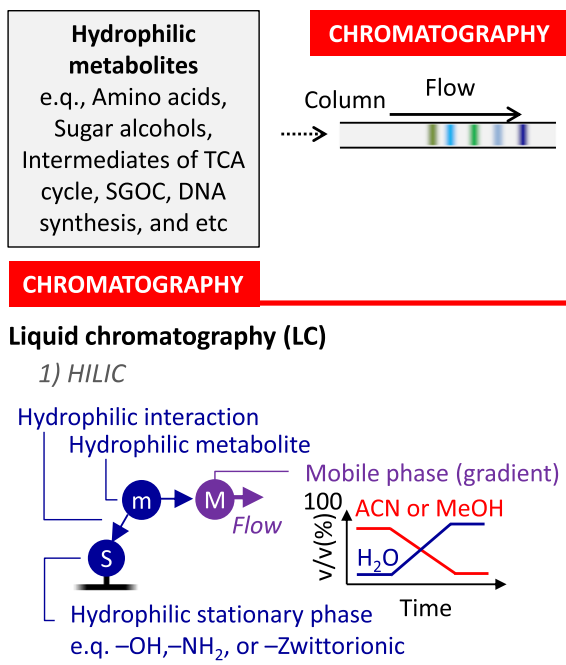

Gas chromatography (GC)

1) Derivatization

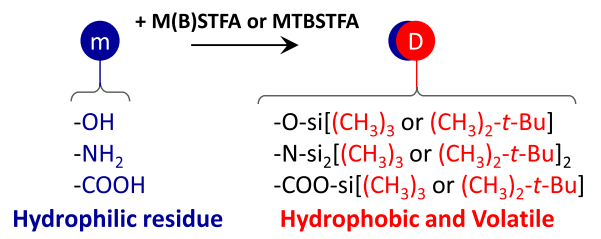

\section{MASS SPECTROMETRY}

GC: EI-MS
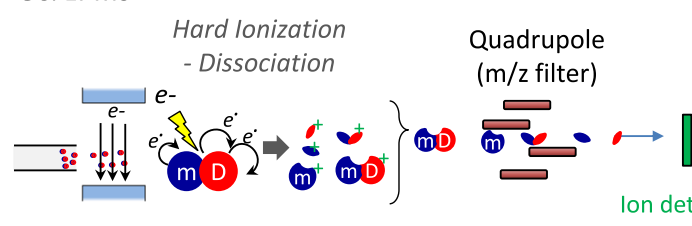

Ion detector

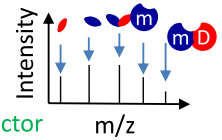

\section{LC: ESI-MS}

Soft Ionization
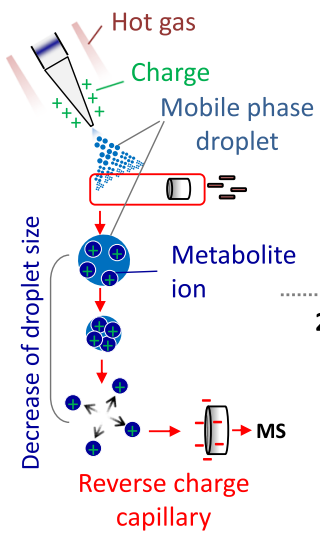

1) Targeted - Triple Q (MS/MS)

non-target ions

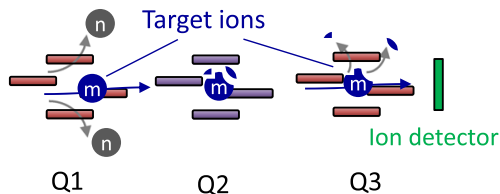

( $\mathrm{m} / \mathrm{z}$ filter) (collision) ( $\mathrm{m} / \mathrm{z}$ filter)

2) Non-targeted: Q-TOF or Q-orbitrap (exactive) High-resolution mass

Quadrupole + Time of Flight or Orbitrap
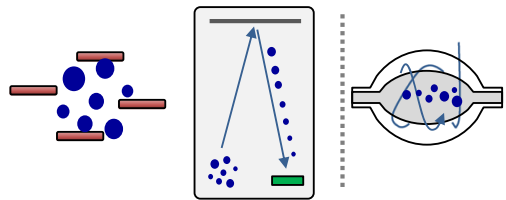

\section{METABOLITE ID}

Intensity $\mathrm{m} / \mathrm{z}$ Intensity $\mathrm{m} / \mathrm{z}$

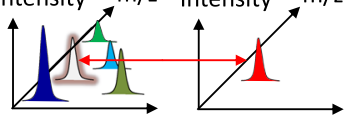

Retention time (RT)

Unknown

STD

\section{) Chromatographic separation in high temperature}

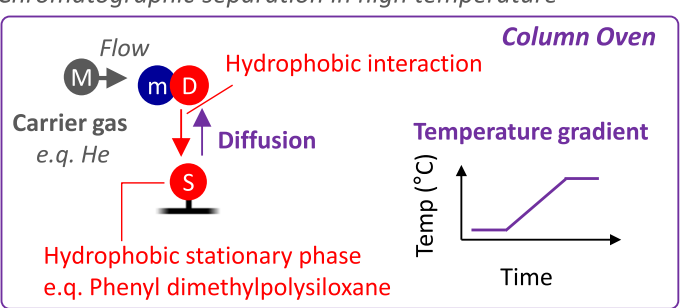

\section{METABOLITE ID}

RT and EI-MS similarity Sample (Unknown) STD MD

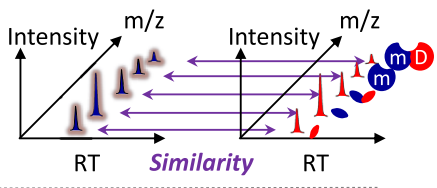

$R T$ and MS/MS based specificity

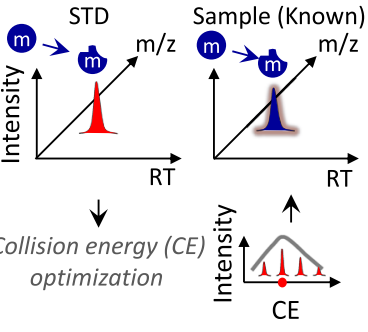

$R T$ and high-resolution $\mathrm{m} / \mathrm{z}$ Intensity $\mathrm{m} / \mathrm{z}$ Intensity $\mathrm{m} / \mathrm{z}$

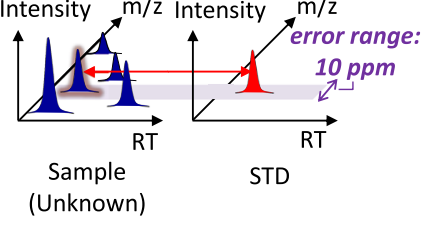

Fig. 1 (See legend on next page.) 
(see figure on previous page)

Fig. 1 Chromatography coupled to mass spectrometry-based metabolite profiling. (Chromatography) The hydrophilic metabolites are separated by chromatography based on their chemical properties. For LC, the types of stationary phase and mobile phase, and the gradient of and composition of the mobile phase must be optimized to facilitate the optimal separation of metabolites of interest. In contrast, prior to GC-based separation, the metabolites must be derivatized to be volatile. Because the volatile-derivatized metabolites only interact with the stationary phase of the column and not the chemically inert gas mobile phase, the column oven temperature gradient needs to be optimized to achieve optimal metabolite separation. (Mass spectrometry) The separated metabolites are ionized and discriminated via mass spectrometry based on their molecular mass to charge ratio $(\mathrm{m} / \mathrm{z})$. The identity of metabolites is further determined by comparing with an authentic standard. (Metabolite ID) LC-MS analysis: after ionization by ESI, the ions with the same retention time can be discriminated via their MS/MS fragmentation (in triple Q) or via highresolution mass accuracy (in Q-TOF or Orbitrap). In contrast, for GC-MS analysis, the metabolite ion specificity is based on their dissociation fragment pattern following El-based hard ionization. TCA tricarboxylic acid, SGOC serine, glycine, and one carbon, LC liquid chromatography, GC gas chromatography, ESI electrospray ionization, El electron (impact) ionization, Q-TOF quadrupole-time of flight, ACN acetonitrile; $\mathrm{MeOH}$ methanol, m/z mass to charge ratio, M(B)STFA N-methyl-N (or N,O-bis)-(trimethylsilyl) trifluoroacetamide, MTBSTFA N-(tert-butyldimethylsilyl)- $N$ -

methyltrifluoroacetamide, RT retention time, CE collision energy

which consists of a wide range of metabolites with diverse chemical characteristics. For example, lipids, which serve as major components of cellular membranes and function as signaling molecules, have chemical properties that require specialized analysis relative to other hydrophilic metabolites. The chloroform/methanol extraction solvent, which is commonly used for lipid extraction ${ }^{26}$, poorly extracts hydrophilic metabolites ${ }^{13}$. Further, the chromatographic conditions that can separate hundreds of different lipid structures that have similar hydrophobic chemical properties that may not efficiently separate hydrophilic compounds ${ }^{27}$. Likewise, mass spectrometrybased lipidomics techniques have been optimized for lipid analysis $^{28}$. Both LC-MS and GC-MS-based approaches have been employed to profile multiple types of fatty acids effectively allowing for the exploration of lipid metabolism in cancer and its association with cancer survival ${ }^{29,30}$. By comparing LC-MS-derived lipid profiles of cell culture medium over time, Kamphorst et al. identified that mutant KRAS drives unsaturated fatty acid uptake, particularly lysophosphatidyl choline (C18:1), to bypass the need for de novo synthesis under nutrient limitation such as in hypoxia ${ }^{31}$. Moreover, Svensson et al., used a GC-MS-based fatty acid profiling approach to measure palmitate and stearate and demonstrated a necessity for de novo fatty acid synthesis in sustaining lung tumor growth, and an associated sensitivity of multiple NSCLC models to acetyl-CoA carboxylase (ACC) inhibition ${ }^{32}$.

\section{Redox couples}

Some metabolic intermediates of pathways implicated in tumorigenesis are not reliably detected by global metabolite profiling approaches due to chemical or biological instability, or because they are conjugated to other types of biomolecules. Previous studies have demonstrated that redox homeostasis is crucial to cancer initiation and progression and that the ratio of glutathione (GSH) and its oxidized form (GSSG) is an important indicator of redox balance ${ }^{33}$. Since GSH is easily oxidized to GSSG during extraction from biological samples, recent studies have described sample preparation methods preventing GSH oxidization of its vulnerable thiol group with the use of derivatization reagents such as 4fluoro-7-sulfamoylbenzofurazan (ABD-F) or $N$-ethyl maleimide during extraction. These methods enable simultaneous and quantitative measurement of GSH and GSSG by $\mathrm{LC}-\mathrm{MS}^{34,35}$, thereby enhancing the study of redox metabolism in cancer. Indeed, ABD-F was employed to quantify the redox potential (GSH/GSSG ratio) in K-Ras mutant (K-Ras ${ }^{\mathrm{G} 12 \mathrm{D} /+}$ ) mouse embryonic fibroblasts. This led to an observed increase in the GSH/ GSSG ratio of K-Ras mutated cells compared to wild-type, which was mediated by the antioxidant response regulator NF-E2-related factor 2 (NRF2) ${ }^{33}$. Recent advances in sample extraction have improved the stability of the $\mathrm{NADP}^{+}: \mathrm{NADPH}$ and $\mathrm{NAD}^{+}: \mathrm{NADH}$ redox couples, which are easily interconverted as well ${ }^{36}$.

\section{One carbon metabolism}

Recent studies have signified the importance of serine, glycine, and one carbon (SGOC) metabolism in cancer progression due to its contribution to glutathione and nucleotide synthesis and the maintenance of DNA methylation ${ }^{37-40}$. Though increased purine and pyrimidine synthesis has been linked directly to active one carbon metabolism ${ }^{38,40}$, we still lack a comprehensive understanding of the intermediate metabolism leading to this increase due to multiple obstacles preventing the quantification of one carbon-bound tetrahydrofolate derivatives (1C-THFs). 1C-THFs are vulnerable to oxidation, exist in cells as polyglutamated forms which obscure chemical structures of each 1C-THF species, and are readily interconverted due to spontaneous chemical or enzymatic reactions during extraction ${ }^{41}$. Recently, Li et al. developed an LC-MS-based analytical method for quantitative determination of $1 \mathrm{C}-\mathrm{THFs}^{41}$. During sample preparation, the application of $\mathrm{NaBD}_{3} \mathrm{CN}$ effectively reduced 5,10-methylene-THF into deuterium labeled 5-methylTHF in a short time $(\sim 5 \mathrm{~min})$. Moreover, the authors discriminated 5,10-methylene THF from THF by 


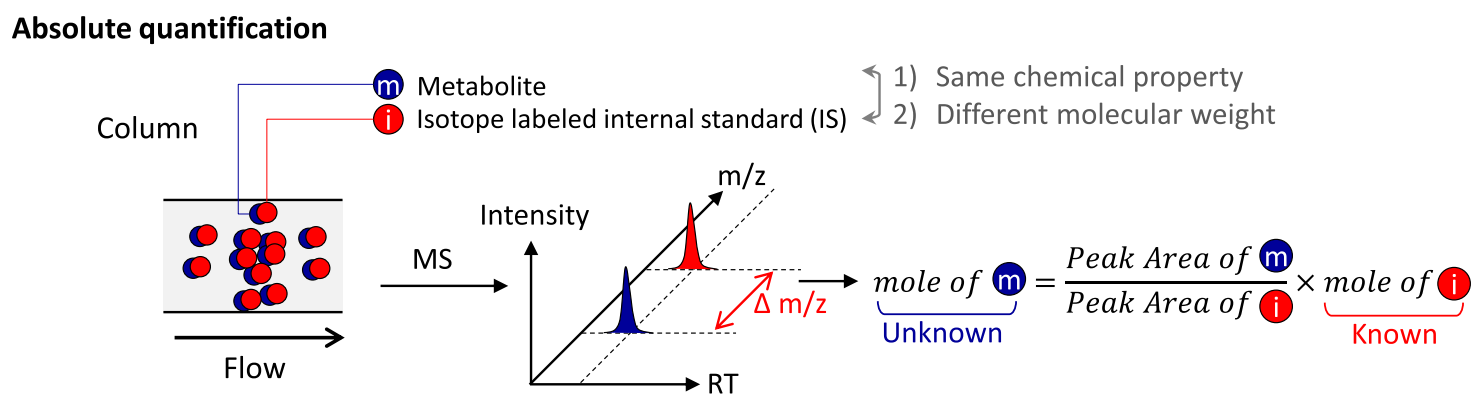

Isotope labeled metabolite tracing

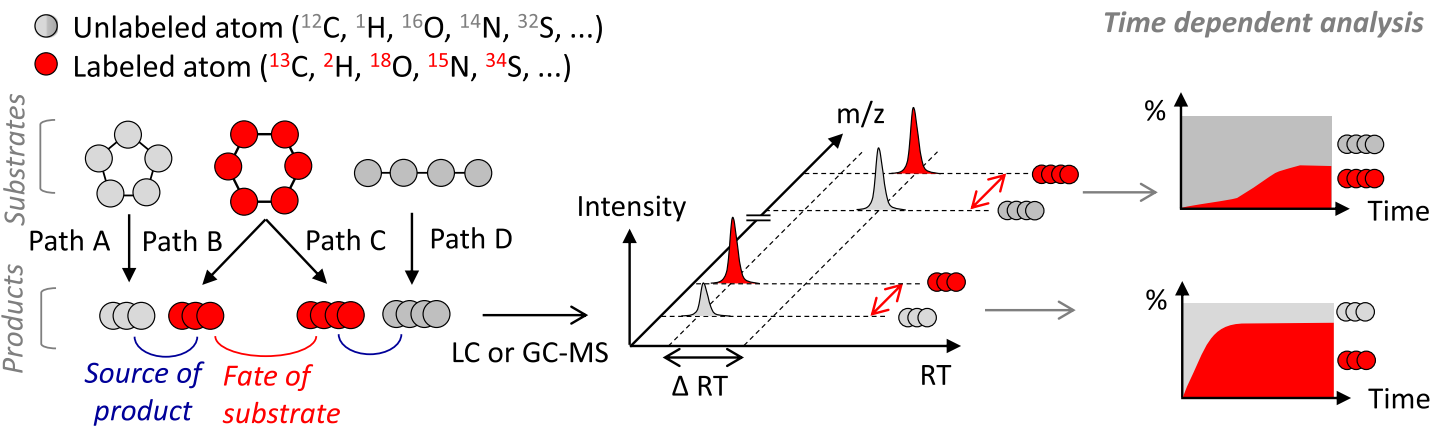

Fig. 2 The use of stable isotopes for absolute quantification and tracing. (Absolute quantification) Isotope-labeled metabolite standards can be used as internal standards (IS) to quantify the unknown amount of metabolites in biological samples. Since the IS has the same chemical properties as the target metabolite, variation during sample preparation and instrumental analysis can be corrected. Furthermore, the metabolite and IS can be distinguished during MS analysis due to the difference in their molecular weights. Finally, the quantity of the metabolite can be calculated by determining the peak area ratio of metabolite to IS, and multiplying by the known amount of the IS. (Metabolite tracing) The fate of isotope-labeled metabolite substrates can be traced into downstream products by chromatography coupled to MS. By calculating the isotope enrichment of a target metabolite, the relative contribution of different pathways to that metabolite pool can be evaluated. Furthermore, time-course analysis facilitates the determination of flux through metabolic pathways. MS mass spectrometry, m/z mass to charge ratio, LC liquid chromatography, GC gas chromatography, RT retention time

preventing their interconversion. Furthermore, by preventing enzymatic interconversion by rapid heating $\left(60^{\circ}\right.$ C, $5 \mathrm{~min}$ ), 5 -formyl-THF could be differentiated from the 5,10-methenyl/10-formyl-THF pool. Finally, the use of folate-free rat serum condensed polyglutamated 1C-THFs into their monoglutamated form, thereby simplifying the chemical diversity of each $1 \mathrm{C}$-THF species. Combined with absolute quantitation and tracing approaches, this method can be applied to both quantify the folate intermediate pool and estimate folate intermediate flux, which will contribute to our understanding of the precise role of one carbon metabolism in cancer ${ }^{42}$.

\section{Absolute quantification}

To conduct mass spectrometry-based absolute quantification of the molar concentration of metabolites in a biological system, stable isotope-labeled internal standards (IS), which have same chemical properties as the target metabolite, are required (Fig. 2). The isotopelabeled internal standard can be used to correct for loss of signal during extraction, fluctuation of signal during mass spectrometry-based analysis, and can also be discriminated from the analyte of interest due to their molecular weight difference. Importantly, the amount of analyte can be quantified based on the peak area ratio of analyte to IS based on the known quantity of the IS. However, due to the expense of stable isotope-labeled IS and the lack of commercially available IS for all metabolites, it is difficult to conduct absolute quantification on the entire cellular metabolome. To overcome this obstacle, Mashego et al., have generated a wide range of stable isotope-labeled metabolites from Saccharomyces cerevisiae by growing them in medium containing only ${ }^{13} \mathrm{C}$ glucose as a nutrient ${ }^{43}$. Since isotopically labeled yeast metabolites can be used as internal standards after their abundance is quantified using non-labeled metabolites, which are relatively inexpensive and easy to obtain, absolute quantification of a range of metabolites is now possible $^{44}$.

Overall, the advances in GC-MS or LC-MS-based nontargeted or targeted metabolite detection have contributed to the discovery and understanding of important metabolic alterations in cancer progression. Furthermore, efforts to refine these techniques are still ongoing, which 
may greatly improve quantitative metabolite coverage and thus the scope of cancer metabolism research. Beyond acquisition of metabolic snapshots, these mass spectrometry-based methods have been applied to trace stable isotope-labeled metabolites for exploring the fate of particular substrates. In the next sections, we will discuss available strategies to interrogate metabolic flux in cancer metabolism.

\section{Metabolite tracing}

The characterization of cancer-associated metabolic phenotypes by global metabolomics analysis has proved essential for establishing the fundamental aspects of cancer metabolism ${ }^{45}$. However robust, these techniques provide a static snapshot of the metabolic activity of tumor cells and are thus incapable of capturing the dynamic nature of cellular metabolism. The use of stable isotope-labeled substrates to trace the isotope enrichment of particular atoms on downstream metabolites permits the determination of the activity of or flux through defined pathways, greatly enhancing the utility of metabolomics analysis ${ }^{46}$ (Fig. 2). Glucose $\left({ }^{13} \mathrm{C}\right)$ and glutamine $\left({ }^{13} \mathrm{C},{ }^{15} \mathrm{~N}\right)$ tracers have been used extensively to demonstrate the importance of central carbon metabolism in meeting the bioenergetic demands of tumors ${ }^{47,48}$. Moreover, this work has illuminated the substantial shunting of glycolytic intermediates into anabolic subsidiary pathways and the engagement of reductive glutamine metabolism to sustain mitochondrial function present in many can$\operatorname{cers}^{38,49}$. However, tracing with these substrates does not support the full interrogation of cancer metabolism.

The recent development of tracing schemes employing a greater variety of labeled substrates has substantially expanded the breadth of metabolism subject to flux analysis (Table 1). For instance, labeled serine and glycine variants have been utilized to demonstrate how serine feeds one carbon metabolism and proliferation ${ }^{40}$. Furthermore, Maddocks et al. demonstrated that serine supports s-adenosylmethionine (SAM) production and the subsequent methylation of nucleic acids in colorectal cancer cells through stimulation of de novo synthesis of adenosine triphosphate (ATP) by tracing dual-labeled ${ }^{13} \mathrm{C},{ }^{15} \mathrm{~N}$-Ser into DNA and RNA ${ }^{50}$.

The study of one-carbon metabolism has also benefitted from the use of deuterium $\left({ }^{2} \mathrm{H}\right)$-based tracers. $\left[2-{ }^{2} \mathrm{H}\right]$-Ser was used to resolve active exchange between serine and glycine as an indicator of the rate of one-carbon metabolism ${ }^{51}$. Moreover, Ducker et al. traced $\left[2,3,3-{ }^{2} \mathrm{H}\right]$-serine into palmitate and proline to differentiate between cytosolic and mitochondrial folate metabolism and show a compensatory exchange between these distinct onecarbon pools when the mitochondrial system was lost in colorectal cancer ${ }^{42}$. Though thought to be functionally separate, this trafficking between compartments was also seen in non-small cell lung cancer (NSCLC) ${ }^{52}$. These studies reveal that compartmentalization not only supports distinct metabolism but also provides an intrinsic safeguard against the collapse of one-carbon metabolism when either system is compromised, indicating an inherent complexity for targeting this network therapeutically. Nevertheless, these substrates can aid in the disentanglement of one-carbon metabolism, allowing for the identification of novel metabolic inputs that drive the formation of one-carbon units, which support protumorigenic maintenance of nucleotide levels, antioxidant capacity and the epigenome ${ }^{53}$.

The advent of ${ }^{2} \mathrm{H}$-tracing has also enhanced the investigation of the fate of the critical redox cofactors nicotinamide adenine dinucleotide $(\mathrm{NAD}[\mathrm{H}])$ and nicotinamide adenine dinucleotide phosphate (NADP $[\mathrm{H}])$. The regeneration of $\mathrm{NAD}^{+}$by lactate dehydrogenase (LDH) supports the robust glycolytic flux hallmark of Warburg metabolism, yet sustained lactate generation from glucose restricts shunting of glycolytic intermediates for biosynthetic metabolism critical to tumor growth ${ }^{54}$. Intriguingly, Hanse et al. used $\left[4-{ }^{2} \mathrm{H}\right]$-glucose to label cytosolic NADH and demonstrated that malate dehydrogenase 1 (MDH1) regenerates $\mathrm{NAD}^{+}$to sustain glycolysis in the presence of biosynthetic diversion of glycolytic intermediates upstream of lactate ${ }^{55} \cdot\left[4-{ }^{2} \mathrm{H}\right]$-glucose was also employed to demonstrate that malate consumption by the cytosolic malic enzyme (ME1) contributes substantially to the NADPH pool in highly lipogenic adipocytes and that this is disrupted under hypoxia, where oxPPP is engaged to sustain NADPH levels ${ }^{56}$. Given the substantial nutrient stresses imposed by the tumor microenvironment, this resolution of NADPH metabolism under varied environmental pressures will greatly enhance the dissection of metabolic rewiring that sustains tumor resilience.

NADPH is critical for anabolic metabolism such as fatty acid synthesis and ${ }^{2} \mathrm{H}$-tracing has been particularly useful for the interrogation of this pathway. The administration of deuterated water $\left(\mathrm{D}_{2} \mathrm{O}\right)$ permits tracing into fatty acids in vivo to assess the rate of de novo fatty acid synthesis ${ }^{57}$. Importantly, $\mathrm{D}_{2} \mathrm{O}$ tracing facilitated the discovery of robust fatty acid biosynthesis in NSCLC described previously $^{32}$. Through establishing the rate of fatty acid synthesis, they were able to monitor a direct phenotypic response to pharmacological ACC inhibition and thus elucidate the mechanism of its anti-cancer activity. This highlights the interrogative power provided by tracing in the characterization of novel therapeutic targets and their associated therapies.

The demand for NADPH is met by flux through various pathways in multiple cellular compartments. As such, significant strides have been made to trace compartmentspecific generation of $\mathrm{NADPH}^{42,52,56}$. The oxidative pentose phosphate pathway (oxPPP) is a major contributor to 
Table 1 Stable isotope-labeled metabolic tracers

\begin{tabular}{|c|c|c|}
\hline Substrate & Target metabolism (indicator metabolite) & References \\
\hline \multirow[t]{3}{*}{${ }^{13} \mathrm{C}-$-Glucose } & - Glycolysis & $38,49,56,117$ \\
\hline & - TCA cycle & \\
\hline & - Biosynthesis: amino acid, lipid, nucleotide & \\
\hline \multirow[t]{2}{*}{${ }^{13} \mathrm{C}-$-Glutamine } & - TCA cycle, & 49,56 \\
\hline & - Biosynthesis: amino acid, lipid, nucleotide & \\
\hline \multirow[t]{2}{*}{${ }^{15} \mathrm{~N}$-Glutamine } & - Biosynthesis: amino acid and nucleotide & 117 \\
\hline & - Urea cycle & \\
\hline \multirow[t]{3}{*}{${ }^{13} \mathrm{C}$-Acetate } & - TCA cycle & 59 \\
\hline & - Lipid synthesis & \\
\hline & - Histone acetylation & \\
\hline \multirow[t]{3}{*}{${ }^{13} \mathrm{C}\left({ }^{15} \mathrm{~N}\right)$-Serine } & - Serine, glycine, and one-carbon metabolism & 50 \\
\hline & - Nucleotide and S-adenosylmethionine & \\
\hline & biosynthesis & \\
\hline$\left[2^{2} \mathrm{H}\right]$-Serine & - Serine, glycine, and one-carbon metabolism & 51 \\
\hline \multirow[t]{2}{*}[2,3,3-{}^{2}\mathrm{H}]{-Serine } & - Serine, glycine, and one-carbon metabolism (palmitate [cytosolic], proline [mitochondrial]) & 42 \\
\hline & - NADPH (thymidine triphosphate) & \\
\hline$\left[1-{ }^{2} \mathrm{H}\right]-$ Glucose & - Oxidative pentose phosphate pathway (NADPH, palmitate) & 56 \\
\hline$\left[3-{ }^{2} \mathrm{H}\right]-$ Glucose & - Oxidative pentose phosphate pathway (NADPH, palmitate) & 52 \\
\hline \multirow[t]{2}{*}[4-{}^{2}\mathrm{H}]{-Glucose } & - NADH (lactate, malate), & $52,55,56$ \\
\hline & - Malic enzyme (malate [cytosolic], NADPH, palmitate) & \\
\hline$\left[2,2,3,3-^{2} \mathrm{H}\right]$-Dimethyl Succinate & - Malic enzyme (malate [mitochondrial], NADPH, palmitate) & 56 \\
\hline $\mathrm{D}_{2} \mathrm{O}$ & - Lipid biosynthesis & 32 \\
\hline
\end{tabular}

the cytosolic NADPH pool ${ }^{58}$, and its contribution to the this pool can be assessed with $\left[3-{ }^{2} \mathrm{H}\right]$ glucose $\mathrm{e}^{52}$. Further, folate metabolism can contribute to both cytosolic and mitochondrial NADPH. Liu et al. used $\left[2,3,3-{ }^{2} \mathrm{H}\right]$ serine to evaluate the total contribution of folate metabolism to cellular NADPH levels. Furthermore, they discriminated between cytosolic and mitochondrial NADPH production by evaluating the labeling of thymidine triphosphate, a concomitant byproduct of this one-carbon metabolism ${ }^{56}$. Additionally, Lewis et al. developed a reporter system based on the mutant IDH (mtIDH1/2) production of 2hydroxyglutarate (2-HG) from alpha-ketoglutarate (a$K G)$, which requires NADPH. Introduction of either the cytosolic mtIDH1 or mitochondrial mtIDH2 promoted labeling of 2-HG from multiple deuterated substrates without apparent interference of normal a-KG metabolism. Using this system, they traced the hydride transfer from deuterated serine to 2-HG to show a mitochondrial predominance for serine conversion to glycine in NSCLC cells ${ }^{52}$. The tandem use of these substrates will permit further interrogation of specific metabolic networks that support biosynthesis in cancer.

Compartment-specific metabolism has also been assayed following cellular fractionation. Through coupling ${ }^{13} \mathrm{C}$-acetate tracing and nuclear isolation, Bulusu et al. show a hypoxia-induced nuclear translocation of acetylCoA synthetase 2 that maintains pro-tumorigenic histone acetylation through the regeneration of acetyl-CoA from endogenous acetate released from histone deacetylation in breast cancer cells ${ }^{59}$. This provided a distinct pool of acetyl-CoA for histone acetylation that preserved exogenous acetate for mitochondrial oxidation or cytosolic fatty acid biosynthesis during nutrient limitation. Moreover, Chen et al. developed a rapid method for the purification of intact mitochondria to quantify matrix metabolites by LC-MS. They show a poor correlation between whole-cell and matrix specific changes in metabolite levels following treatment with electron transport chain inhibitors, particularly the NADH:NAD ${ }^{+}$and GSH: GSSG redox couples ${ }^{60}$. This demonstrates a whole cell capacity to buffer specific metabolic dysregulation and the 
need to differentiate the compartmentalized effects of cellular stress, such as that induced by anti-cancer therapeutics, on metabolism.

\section{In vivo studies of metabolism}

Though these studies demonstrate the investigative power provided by metabolic flux analysis at the bench, a full elucidation of the metabolic derangements that support tumor growth and progression requires the direct study of the disease at the patient level. Clinical evaluation of tumor metabolism is an established practice, as the fundamental diagnostic technique, 18Ffluorodeoxyglucose positron emission tomography (FDGPET) is dependent on Warburg metabolism and the propensity for tumors to take up glucose at a higher rate than adjacent normal tissue ${ }^{61}$. Other radiolabeled metabolites have been used to demonstrate basic metabolic phenotypes of tumors such as amino acid transport or regionalized hypoxia ${ }^{61,62}$. Hyperpolarized ${ }^{13} \mathrm{C}$-metabolite infusions have been performed for more sophisticated MR-based tumor detection and grading, however the technical advances described here are being translated to patient populations to allow for the capture of metabolic flux with isotope-labeled substrates ${ }^{62-65}$.

Hensley et al. assessed the metabolic profile of lung tumors from nine NSCLC patients who received ${ }^{13} \mathrm{C}$ glucose infusions prior to tumor resection and showed substantial intratumoral heterogeneity in glucose metabolism, with concurrent glycolysis and glucose oxidation. Through the integration of preoperative FDG-PET and dynamic contrast-enhanced magnetic resonance imaging (DCE-MRI), they also demonstrated that regional glucose metabolism correlated with tumor perfusion. Most strikingly, examination of the fractional enrichment of glycolytic and TCA cycle intermediates indicated lactate utilization as a carbon source, which they recapitulated through $\left[2-{ }^{13} \mathrm{C}\right]$ lactate tracing in mouse xenografts derived from NSCLC cell lines harboring KRAS or EGFR mutations ${ }^{66}$.

In a cohort of early-stage lung cancer patients, the preoperative infusion of ${ }^{13} \mathrm{C}$-glucose led to the discovery of enhanced pyruvate carboxylase (PC) flux in lung tumors relative to benign lung, which correlated with increased PC expression ${ }^{67}$. The resected tumors were then sectioned and labeled with ${ }^{13} \mathrm{C}$-glucose ex vivo to examine downstream metabolism ${ }^{68}$. The cultured tumor sections retained the enhanced PC flux seen in the intact tumors and further interrogation of NSCLC cell lines revealed a requirement for PC activity in maintaining proliferation. This ex vivo system is compatible with complex tracing not currently optimized for the clinic in the presence of an intact tumor microenvironment, thus more physiologically relevant than two- or threedimensional culture. Collectively, these studies demonstrate that lung tumors demonstrate significantly more mitochondrial carbon metabolism than previously thought based on cell culture studies and that the microenvironment and tumor perfusion have a significant impact on tumor metabolism. Further, they emphasize the need to perform in vivo assays of cellular metabolism.

\section{Metabolic sensors}

Although the advances in sample preparation and instrumentation have dramatically increased the metabolite coverage provided by metabolomics analysis, these techniques require extended run-up times prior to the detection of metabolite levels which limits the practical number of samples suitable for analysis in each experimental run, thus the number of observable time points for an experiment is restricted when multiple time points are required to assess metabolic flux. Unfortunately, since much of metabolism occurs very rapidly, the limited number of sampling time points may prevent the observation of important metabolic alterations in cancer. To overcome these limitations, multiple genetically encoded biosensors have been developed to facilitate the real-time detection of metabolic indicators (Fig. 3).

\section{Glucose}

Biosensors have improved the evaluation of dynamic flux and metabolite exchange between cells and their environment ${ }^{69,70}$. Takanaga et al. improved upon an existing genetically encoded fluorescence resonance energy transfer (FRET)-based nanosensor to monitor glucose influx ${ }^{71}$. They employed a construct based on the resonance energy transfer between cyan and yellow fluorescent proteins that flank a bacterial glucose binding protein, where glucose binding promotes a conformational change that brings the fluorescent proteins closer in proximity thus increasing the FRET signal. To optimize the biosensor for physiological glucose concentrations, they reduced the space between the fluorophores and the overall flexibility of the sensor. Furthermore, Bittner et al. extended the utility of FRET-based glucose sensing to measure glycolytic flux by establishing the rate of cytosolic glucose depletion ${ }^{69}$. This application was subsequently implemented to assess the rates of glucose depletion as well as lactate production via the lactate biosensor, Laconic, to indicate glycolytic metabolism in response to hypoxia in breast cancer cells. The real-time monitoring of lactate production led to the characterization of a novel non-catalytic function of the hypoxiainduced carbonic anhydrase IX in facilitating the rapid efflux of lactate via monocarboxylate transporters, which was required to sustain viability during hypoxic stress ${ }^{70}$. Similar FRET-based sensors that capitalize on the metabolite binding properties of bacterial or yeast proteins have been developed for the measurement of other 


\section{A. FRET}

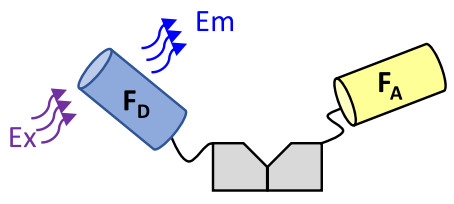

Metabolite
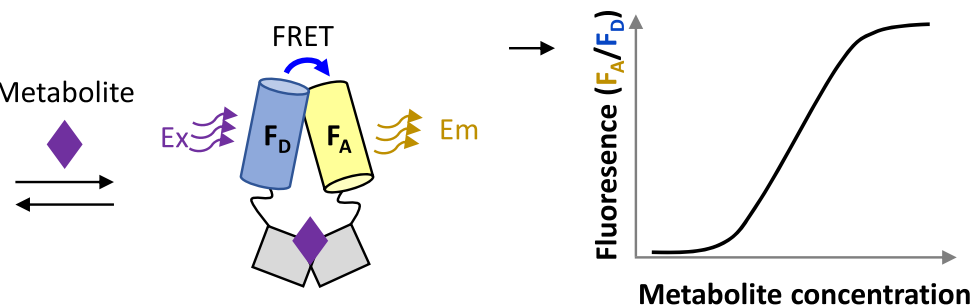

B. Ratiometric

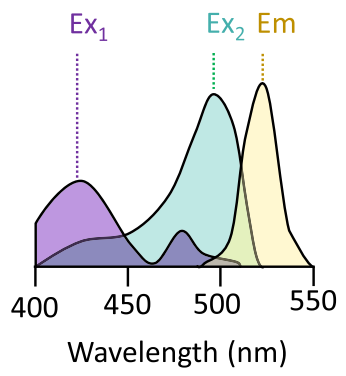

- Metabolite A

bolite B
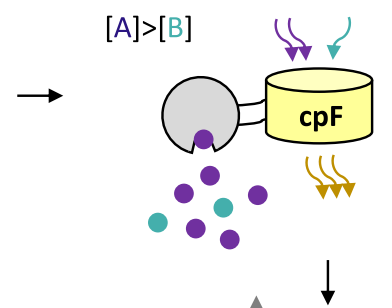

$[\mathrm{A}]<[\mathrm{B}]$
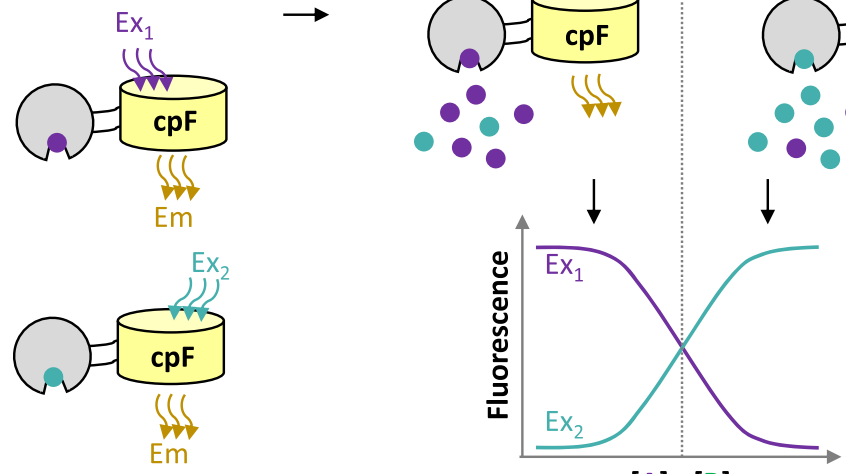

$[\mathrm{A}]:[\mathrm{B}]$

Fig. 3 Genetically encoded fluorescent biosensors facilitate dynamic monitoring of metabolism. a (FRET) Many genetically encoded fluorescent biosensors are based on the FRET between donor $\left(F_{D}\right)$ and acceptor $\left(F_{A}\right)$ fluorophores flanking a bacterial or yeast metabolite-binding domain. In the absence of metabolite binding, excitation of the $F_{D}$ results in the emission of its associated fluorescence. However, when the metabolite binds, the biosensor undergoes a conformational change that permits FRET between fluorophores and emission of $F_{A}$ fluorescence. The $F_{A}: F_{D}$ fluorescence ratio is thus indicative of the intracellular metabolite concentration. $\mathbf{b}$ (Ratiometric) Circularly permutated fluorophores associated with a metabolite-binding domain that recognizes two distinct but structurally similar metabolites can be employed for the ratiometric detection of these metabolite pairs (A \& B). These fluorophores have two distinct excitation wavelengths $\left(E x_{1} \& E x_{2}\right)$ that stimulate fluorescence of the same emission wavelength (Em). Occupancy of metabolite A increases the fluorescent signal associated with $E x_{1}$ and vice versa, so that the fluorescence ratio of $\mathrm{Ex}_{1}: \mathrm{Ex}_{2}$ is representative of the ratio of metabolite $\mathrm{A}$ : metabolite $B$

metabolite fluxes, including mitochondrial pyruvate uptake $\mathrm{e}^{72,73}$, changes in the ATP:ADP ratio ${ }^{74}$, and glutamate $^{75}$, phosphate ${ }^{76}$ and tryptophan transport ${ }^{77}$ (Table 2).

\section{$\mathrm{pH}$}

The robust production and export of lactate by many tumors is associated with the acidification of the microenvironment, which disrupts the function of tumor associated stromal cells while enhancing the aggressiveness of neoplastic cells ${ }^{78}$. To sustain a $\mathrm{pH}$ gradient that favors the simultaneous release of lactate and protons from cancer cells, intracellular $\mathrm{pH}\left(\mathrm{pH}_{\mathrm{i}}\right)$ is tightly maintained within a narrow range of 7.2-7.4 $4^{79,80}$. To assess fluctuations in $\mathrm{pH}_{\mathrm{i}}$, Matlashov et al. developed a genetically encoded fluorescent $\mathrm{pH}$ sensor (SypHer-2) that can be used for the ratiometric determination of $\mathrm{pH}_{\mathrm{i}}$. SypHer2 is structurally based on the $\mathrm{pH}$-sensitive HyPer biosensor for $\mathrm{H}_{2} \mathrm{O}_{2}$ that has been mutated to make it insensitive to $\mathrm{H}_{2} \mathrm{O}_{2}$ as well as enhance its fluorescence intensity in response to changes in $\mathrm{pH}_{\mathrm{i}}^{81}$. Shirmanova et al. demonstrated utility in both monolayer and 3D culture systems, where they observed a $\mathrm{pH}$ gradient in spheroids with decreasing $\mathrm{pH}$ towards the core. Moreover, they successfully extended the use of SypHer-2 to a xenograft model of cervical cancer, showing an overlap of acidic and hypoxic tumor regions ${ }^{80}$.

In a subsequent study, Shirmanova et al. employed SypHer-2 to show that cisplatin treatment induces intracellular acidification in cervical cancer cells. They found that those cells exhibiting persistent acidification die, whereas cells that can recover a more alkaline $\mathrm{pH}_{\mathrm{i}}$ due to a shift towards mitochondrial oxidation survive, revealing that metabolic flexibility contributes to cancer cell persistence in response to prolonged exposure to anti-cancer therapies ${ }^{82}$. Moreover, this demonstrates the value of dynamic biosensors in the characterization of intrinsic metabolic responses to therapy, which may uncover potential biomarkers for the assessment of therapeutic efficacy as well as inform the development of rational combinations to exploit vulnerabilities imposed by monotherapy. 
Table 2 Genetically encoded fluorescent biosensors

\begin{tabular}{|c|c|c|c|c|}
\hline Sensor & Type & Fluorophore(s) (Ex/Em) & Target & References \\
\hline \multirow[t]{2}{*}{ FLII ${ }^{12} \mathrm{Pglu}-70086$} & \multirow[t]{2}{*}{ Hetero-FRET } & Donor: eCFP (408/485) & \multirow[t]{2}{*}{ Glucose } & \multirow[t]{2}{*}{71} \\
\hline & & Acceptor: Citrine-eYFP (525/550) & & \\
\hline \multirow[t]{2}{*}{ Laconic } & \multirow[t]{2}{*}{ Hetero-FRET } & Donor: mTFP (462/492) & \multirow[t]{2}{*}{ Lactate } & \multirow[t]{2}{*}{70} \\
\hline & & Acceptor: Venus (515/528) & & \\
\hline \multirow[t]{2}{*}{ Pyronic } & \multirow[t]{2}{*}{ Hetero-FRET } & Donor: mTFP (462/492) & \multirow[t]{2}{*}{ Pyruvate } & \multirow[t]{2}{*}{72} \\
\hline & & Acceptor: Venus (515/528) & & \\
\hline \multirow[t]{2}{*}{ FLIPE } & \multirow[t]{2}{*}{ Hetero-FRET } & Donor: eCFP (408/485) & \multirow[t]{2}{*}{ Glutamate } & \multirow[t]{2}{*}{75} \\
\hline & & Acceptor: Venus (515/528) & & \\
\hline \multirow[t]{2}{*}{ FLIPPI } & \multirow[t]{2}{*}{ Hetero-FRET } & Donor: eCFP (408/485) & \multirow[t]{2}{*}{ Phosphate } & \multirow[t]{2}{*}{76} \\
\hline & & Acceptor: Venus (515/528) & & \\
\hline \multirow[t]{2}{*}{ FLIPW } & \multirow[t]{2}{*}{ Hetero-FRET } & Donor: eCFP (408/485) & \multirow[t]{2}{*}{ Tryptophan } & \multirow[t]{2}{*}{77} \\
\hline & & Acceptor: Venus (515/528) & & \\
\hline \multirow[t]{2}{*}{ Apollo-NADP ${ }^{+}$} & \multirow[t]{2}{*}{ Homo-FRET } & \multirow[t]{2}{*}{ Donor/Acceptor: Venus (515/528) } & NADPH: & \multirow[t]{2}{*}{86} \\
\hline & & & $\mathrm{NADP}^{+}$ & \\
\hline PercevalHR & Ratiometric & Fluorophore: cpGFP (Ex1-430, Ex2-490/520) & ATP:ADP & 74 \\
\hline SypHer-2 & Ratiometric & Fluorophore: cpYFP (Ex1-420, Ex2-500/528) & Intracellular pH & 80,81 \\
\hline \multirow[t]{2}{*}{ Peredox } & \multirow[t]{2}{*}{ Ratiometric } & Fluorophore A: cpT-Sapphire (400/510) & $\mathrm{NADH}:$ & \multirow[t]{2}{*}{83,84} \\
\hline & & Fluorophore B: mCHERRY (587/610) & $N A D^{+}$ & \\
\hline HyPer & Ratiometric & Fluorophore: cpGFP (Ex1-420, Ex2-500/528) & $\mathrm{H}_{2} \mathrm{O}_{2}$ & 86 \\
\hline
\end{tabular}

\section{Redox couples}

The redox metabolism that sustains tumor growth is greatly influenced by the oxidation state of the cell. Though it is now possible to reliably quantify the ratio of important redox couples (GSH:GSSG, NADH:NAD ${ }^{+}$, NADPH:NADP ${ }^{+}$) through the techniques described, realtime detection methods provide greater resolution of the dynamic fluctuations in these pairs and their contribution to redox homeostasis.

To circumvent traditional indirect and static measures of the cellular $\mathrm{NADH}: \mathrm{NAD}^{+}$ratio such as determining the ratio of lactate:pyruvate, Hung et al. developed a genetically encoded ratiometric biosensor based on the green fluorescent protein (GFP) mutant T-Sapphire conjugated to a bacterial NADH-binding protein (Peredox), such that Peredox binding of NADH is associated with increased green fluorescence, permitting single-cell detection of the cytosolic NADH:NAD + ratio $^{83}$. Peredox was used to evaluate glycolytic activity by $\mathrm{Hu}$ et al. in the characterization of PI3K-mediated coordination of actin cytoskeletal dynamics, which promoted release and activation of aldolase and enhanced glycolytic metabo$\operatorname{lism}^{84}$. This study revealed an AKT-independent role of PI3K in the regulation of aerobic glycolysis, thus complicating the therapeutic targeting of the PI3K axis ${ }^{85}$.
To detect changes in the NADPH:NADP ${ }^{+}$ratio, Cameron et al. engineered a HomoFRET biosensor for the detection of $\mathrm{NADP}^{+}$based on glucose-6-phosphate dehydrogenase (G6PD) homodimerization in response to $\mathrm{NADP}^{+}$levels (Apollo-NADP $\left.{ }^{+}\right)^{86}$. HomoFRET is not dependent on a change in fluorescence color but rather the change in fluorescence emission polarization (animosity). They employed Apollo-NADP ${ }^{+}$to show an increase in Apollo-NADP ${ }^{+}$animosity in beta cells following glucose supplementation consistent with stimulation of the oxPPP and NADPH production. Moreover, because Apollo-NADP ${ }^{+}$has a single fluorescence color design, it is compatible with other sensors for simultaneous multiparametric imaging. To demonstrate this, they show that during diamide treatment, NADPH depletion and $\mathrm{H}_{2} \mathrm{O}_{2}$ accumulation, detected via $\mathrm{HyPer}$, do not occur simultaneously. Rather, NADPH depletion preceded any detectable increase in HyPer signal. This combinatory application of Apollo-NADP ${ }^{+}$may be employed to more discretely characterize cancer cell responses to prooxidant therapies.

\section{Seahorse}

In addition to these genetically encoded biosensors that support the surveillance of intracellular metabolism, the 
development of the high-throughput Seahorse extracellular flux analyzer (Agilent Technologies) has been pivotal to the characterization of cancer metabolism ${ }^{87}$. The Seahorse system permits the simultaneous assessment of glycolytic and oxidative metabolism through the coupled sensing of oxygen consumption and extracellular acidification $^{88}$. This provides a platform for the phenotypic evaluation of cancer cells in response to different metabolic substrates or inhibitors ${ }^{89}$. Furthermore, to provide greater resolution of mitochondrial function, a Seahorse-based method has been developed to assess the activity of components of the electron transport chain (ETC) or their associated supercomplexes through the application of specific metabolic substrate/inhibitor combinations in permeabilized cells ${ }^{90}$. While most studies using Seahorse technology use cultured cells, analysis of glycolytic and oxidative metabolism can also be performed on fresh tumor tissue ${ }^{91}$. Using the Seahorse XF Analyzer, Miller et al. observed an increase in both oxygen consumption and extracellular acidification in colorectal adenocarcinoma compared to healthy tissue ${ }^{91}$.

\section{Multidisciplinary approaches to study metabolism}

Cancer metabolism has been evaluated not only with metabolomics technologies, but also with other multidisciplinary approaches. In this section, we will discuss how combining metabolomics with other technical approaches has led to greater insight into cancer metabolism.

\section{Multi-omics approaches}

Metabolic networks are complex systems responsive to multiple layers of molecular regulation. Therefore, great insight into cancer metabolism has been gleaned from the integration of multiple "-omics" technologies, which allows for the identification of metabolic dysregulation at the genome, proteome, and metabolome level simultaneously. For example, Kottakis et al. applied both transcriptional and proteomics approaches to supplement metabolomics analysis in the evaluation of metabolic alterations associated with the loss of LKB1 in KRAS mutant pancreatic ductal cells $(\mathrm{KL})^{92}$. Coordinated gene set enrichment analysis and serine/glycine labeling from ${ }^{13} \mathrm{C}$ glucose tracing indicated increased SGOC metabolism in $\mathrm{KL}$ cells ${ }^{92}$. Moreover, proteomics analysis showed that SAM-dependent enzymes were increased in KL cells, which was associated with significantly higher DNA methylation relative to KRAS-mutant cells. Furthermore, these cells exhibited a greater sensitivity to the inhibition of serine synthesis and DNA methylation indicating a role for activated serine metabolism in supplying SAM for DNA methylation and revealing a therapeutic vulnerability in pancreatic cancer ${ }^{92}$.
Interestingly, Kim et al. performed complementary transcriptomic and metabolomic analyses to uncover an alternative metabolic circuit for sustaining nucleotide levels independent of SGOC metabolism in KL-NSCLC cells $^{93}$. They observed an increase in urea cycle intermediates, including arginine, coupled with the accumulation of transcripts for the urea cycle rate-limiting enzyme, carbamoyl phosphate synthetase-1 (CPS-1). Through subsequent targeting of CPS-1, they demonstrated that these KL cells rely on the urea cycle to drive pyrimidine synthesis from ammonia, which may serve as a metabolic vulnerability to target therapeutically. Similarly, we integrated gene expression profiles and serine labeling patterns from ${ }^{13} \mathrm{C}$-glucose in a panel of human NSCLC cell lines to identify the transcription factor NRF2 as a driver of serine biosynthesis in NSCLC. Further, NSCLC cells with aberrant NRF2 activity were dependent on enhanced serine synthesis for glutathione and nucleotide production, suggesting a potential therapeutic target for NSCLC with NRF2 activation ${ }^{38}$.

As evidenced above, multi-omic integration is a powerful tool for identifying metabolic alterations and elucidating their function in cancer progression. Importantly, recent advances in sequencing technologies have facilitated singe cellular genome sequencing in a highthroughput manner, enabling the delineation of genetic heterogeneity between cells within a tumor ${ }^{94-97}$. Likewise, single cellular proteomics and metabolomics approaches are under development ${ }^{98-104}$. Although the sensitivity of mass spectrometry-based analytical technologies has been greatly improved to detect trace levels of proteins or metabolites, measurement of intact proteins or metabolites from single cells without alteration of their molecular structure during sample preparation is still challenging ${ }^{105,106}$. To address this problem, Miller et al. optimized enzyme histochemistry on frozen tissue from cancer patients to examine the activity of metabolic enzymes involved in carbon metabolism at a single-cell resolution $^{91}$. By combining activity assays with immunostaining for cell type markers, they could determine enzyme activity in discrete populations. However, this technique is does not assay global metabolism in a highthroughput manner and once optimized, multi-omics analysis of single cells will be critical to understanding inter-cellular heterogeneity within the complex tumor microenvironment ${ }^{107}$.

\section{Genome editing}

Integrative "-omics" approaches are effective tools for observing existing perturbations of complex biological systems such as metabolism. These tools have often been employed following targeted manipulations of the genome to investigate the functional role of a gene in a particular phenotype. Although the advantages of targeted 


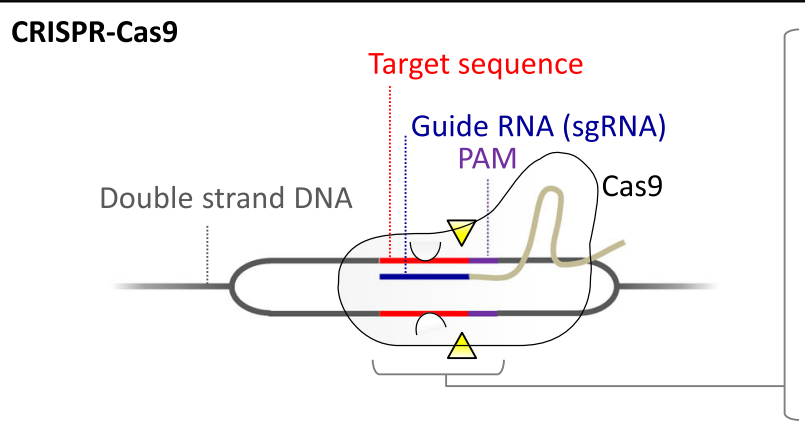

CRISPR-Cas9 based loss-of-function screening
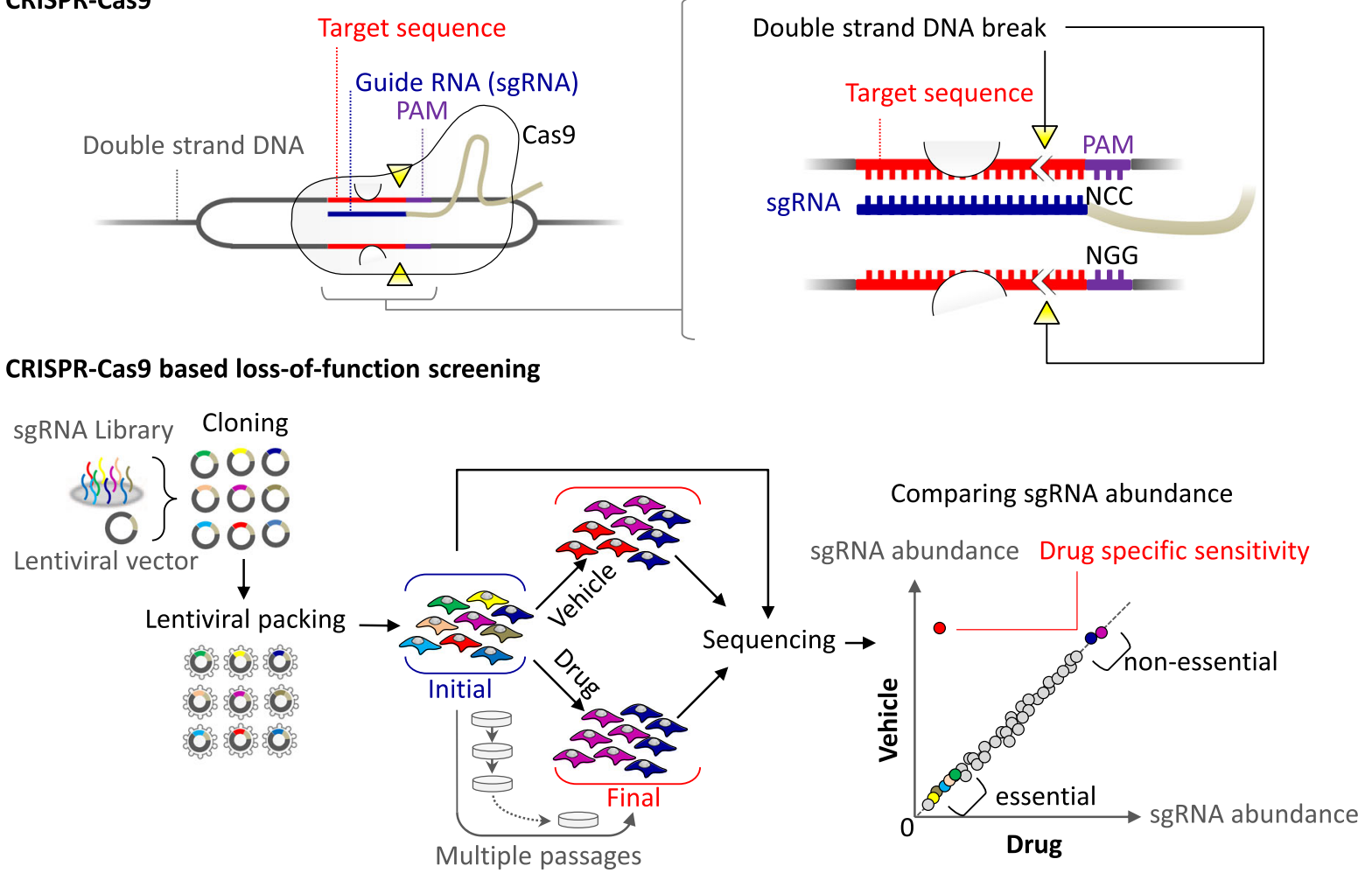

Fig. 4 CRISPR/Cas9-based screening for the study of metabolism. (CRISPR-Cas9) The CRISPR/Cas9 system induces DNA double strand breaks at target genes of interest, thereby inducing gene knockouts. Cas9 specificity is derived from the complementarity of the single-guide RNA (sgRNA) sequence to the target gene of interest, as well as the requirement of the PAM sequence DNA binding and cleavage. (CRISPR/Cas9-based loss-offunction screening) The CRISPR/Cas9 system can be applied to whole genome loss-of-function screening to identify metabolic genes that are essential under defined metabolic states. First, a library of sgRNAs targeting the whole human genome are cloned into a to lentiviral vector encoding Cas9, and followed by packaging into lentiviral particles. Following infection, cells are treated with vehicle or a metabolic inhibitor, serially passaged, and sequenced. By comparing the abundance of sgRNAs in the final cell populations (both vehicle or inhibitor) to their initial representation, the selective depletion of sgRNAs in the inhibitor treated population can be determined. CRISPR clustered regularly interspaced short palindromic repeats, PAM protospacer adjacent motif, sgRNA single-guide RNA

gene manipulation have been demonstrated extensively, the simultaneous manipulation of numerous genes in a high-throughput manner has not been easily achieved. However, the recent development of clustered regularly interspaced short palindromic repeats (CRISPR) and CRISPR associated protein 9 (Cas9)-based gene knock out (KO) facilitates genome-wide loss of function screening $^{108-110}$ (Fig. 4). The CRISPR-Cas9 system has been successfully employed to determine gene essentiality for cell survival or anti-cancer therapy resistance ${ }^{109,110}$.

Birsoy et al. applied this screening technique to evaluate the metabolic role of the electron transport chain (ETC) in cellular proliferation ${ }^{111}$. They generated and screened a CRISPR guide RNA library targeting thousands of metabolic genes in T-cell leukemia to identify genes associated with ETC function. Through this screen, they identified GOT1-dependent aspartate production to be essential for cancer-cell proliferation in the absence of functional $\mathrm{ETC}^{111}$. In a similar fashion, Ducker et al. performed metabolomics analysis following CRISPR editing of SGOC metabolism $^{42}$. Through this combinatorial approach, they discovered a compensatory role of mitochondrial SGOC metabolism when the cytosolic system is compromised ${ }^{42}$. Notably, this result suggests that targeting SGOC metabolism in only one compartment will be insufficient for cancer treatment.

As with other aspects of tumor biology, the study of cancer metabolism has benefited greatly from the use of genetically engineered mouse models (GEMMs) ${ }^{112}$. Conveniently, the CRISPR/Cas9 system can be applied to genetically edit GEMMs to study the functional role of metabolic genes in vivo ${ }^{3,113-115}$. Recently, Davidson et al. used CRISPR/Cas9 to study metabolic dependencies in multiple KRAS-driven NSCLC mouse models ${ }^{3}$. Surprisingly, they showed that these lung tumors persisted following deletion of glutaminase, suggesting that they are not dependent on enhanced glutamine metabolism, in contrast to what has been commonly observed in vitro 
with NSCLC cell lines. This highlights the importance of the extracellular environment on the metabolic phenotype of cancer cells.

In contrast, the CRISPR-Cas9 mediated deletion of KEAP1 in a mouse model of KRAS-driven lung adenocarcinoma to activate NRF2 resulted in a marked dependence on glutaminase activity ${ }^{115}$, signifying the influence of co-occurring mutations on the divergence of metabolic phenotypes among tumors grouped based on a given genetic alteration. As such, the CRISPR-Cas9 system can be employed to generate multiple GEMMs with a particular mutational background to evaluate differential changes in metabolism driven by the presence of commonly co-occurring alterations. These applications greatly enhance the study of metabolic dysregulation in cancer, especially when combined with metabolomics analyses.

\section{Ex vivo tumor models}

It is well appreciated that traditional cell culturing methods have distinct effects on cellular function relative to the in vivo setting ${ }^{116}$. As in vitro systems have become more sophisticated to better model physiological conditions, there is still a significant need to recapitulate the metabolic milieu ${ }^{117}$. To address this, Cantor et al. developed culture media that corrected the nutrient concentrations of traditional synthetic media to accurately represent those of human plasma (Human Plasma-Like Medium; HPLM). The application of HPLM resulted in significant metabolic alterations relative to the traditional media including an inhibition of de novo pyrimidine synthesis. This led to the discovery of novel inhibitory activity of uric acid towards uridine monophosphate synthase and a desensitization of cancer cells grown in HPLM to the pyrimidine synthesis inhibitor, 5fluorouracil ${ }^{117}$.

To better replicate the nutrient-liming environment faced by tumors, Birsoy et al. developed a continuous-flow apparatus to circulate media over cultured cells, allowing for the assessment of nutrient depletion over an extended period of time due to the maintenance of particular metabolites at very low concentrations ${ }^{118}$. They employed this system to determine the competitive fitness of a panel of cell lines cultured simultaneously in glucose-depleted media and found that cells harboring deficiencies in oxidative capacity had compromised fitness in the presence of low glucose. This reduced fitness corresponded with increased sensitivity to the biguanide class of mitochondrial inhibitors. These studies demonstrate the importance of establishing physiologically relevant culture conditions for the characterization of cancer metabolism and associated therapeutic sensitivities.

Two-dimensional culture does not recapitulate all of the features of tissues in vivo, including tissue organization, stiffness, and metabolite and oxygen gradients. The Clevers group and others have developed three-dimensional organoid culture systems that more faithfully replicate these tissue features in an in vitro system ${ }^{119}$. To date, tissue-derived organoid lines have been generated from multiple types of human cancer and matched normal tissues $^{120-122}$. The metabolic differences between twodimensional and three-dimensional cultures are under investigated, but optical metabolic imaging of primary breast cancer organoids was found to predict response to clinically relevant anticancer drugs ${ }^{123}$. Thus, the organoid culture system will be a valuable tool to characterize patient-specific cancer metabolism to facilitate personalized therapy targeting metabolism.

\section{Concluding remarks}

Significant advances in analytical methods that support the investigation of cellular metabolism have furthered the discovery and characterization of metabolic programs critical to tumorigenesis. Optimization of MS-based metabolite profiling techniques has greatly expanded the coverage of detectable metabolites, while the emergence of stable isotope tracing and intra- or extracellular metabolic sensors has facilitated the measurement of pathway engagement and compartmental exchange of metabolites. Furthermore, the integration of metabolomics analysis with established -omics platforms has uncovered biology underlying the metabolic rewiring seen in tumors, whereas sophisticated and high-throughput manipulation of the cancer genome with the CRISPR-Cas9 system has revealed novel metabolic vulnerabilities to target therapeutically. Despite these technical developments, further refinement is required to fully quantify the expanse of cellular metabolism in real-time. A continued focus on accurately recapitulating the microenvironment present in intact tumors will be particularly important for furthering our understanding of cancer metabolism. As such, the recent development of patient tumor derived organoid modeling, ex vivo metabolite tracing and HPLM will likely drive future discovery and enhance therapeutic development.

\footnotetext{
Acknowledgements

We apologize to the authors whose work we could not cite due to space limitations. G.M.D. is supported by the Pancreatic Cancer Action Network Pathway to Leadership Award (14-70-25-DENI), the American Cancer Society's Institutional Research Grant, the Miles for Moffitt Milestone Award, and the American Lung Association Lung Cancer Discovery Award (498544). G.M.D. is supported by the Pancreatic Cancer Action Network Pathway to Leadership Award (14-70-25-DENI), the American Cancer Society's Institutional Research Grant, the Miles for Moffitt Milestone Award, and the American Lung Association Lung Cancer Discovery Award (498544).
}

Conflict of interest

The authors declare that they have no conflict of interest. 


\section{Publisher's note}

Springer Nature remains neutral with regard to jurisdictional claims in published maps and institutional affiliations.

Received: 24 October 2017 Accepted: 12 December 2017. Published online: 16 April 2018

\section{References}

1. Warburg, O. Über den Stoffwechsel der Carcinomzelle. Naturwissenschaften 12, 1131-1137 (1924).

2. DeNicola, G. M. \& Cantley, L. C. Cancer's fuel choice: new flavors for a picky eater. Mol. Cell 60, 514-523 (2015).

3. Davidson, S. M. et al. Environment impacts the metabolic dependencies of Ras-driven non-small cell lung cancer. Cell Metab. 23, 517-528 (2016).

4. Luengo, A., Gui, D. Y. \& Vander Heiden, M. G. Targeting metabolism for cancer therapy. Cell Chem. Biol. 24, 1161-1180 (2017).

5. Kimball, E. \& Rabinowitz, J. D. Identifying decomposition products in extracts of cellular metabolites. Anal. Biochem. 358, 273-280 (2006).

6. Prasad Maharjan, R. \& Ferenci, T. Global metabolite analysis: the influence of extraction methodology on metabolome profiles of Escherichia coli. Anal. Biochem. 313, 145-154 (2003).

7. Lu, W., Kimball, E. \& Rabinowitz, J. D. A high-performance liquid chromatography-tandem mass spectrometry method for quantitation of nitrogen-containing intracellular metabolites. J. Am. Soc. Mass Spectrom. 17, 37-50 (2006).

8. Want, E. J. et al. Global metabolic profiling of animal and human tissues via UPLC-MS. Nat. Protoc. 8, 17-32 (2013).

9. Villas-Bôas, S. G., Højer-Pedersen, J., Åkesson, M., Smedsgaard, J. \& Nielsen, J. Global metabolite analysis of yeast: evaluation of sample preparation methods. Yeast 22, 1155-1169 (2005).

10. Yuan, M., Breitkopf, S. B., Yang, X. \& Asara, J. M. A positive/negative ionswitching, targeted mass spectrometry-based metabolomics platform for bodily fluids, cells, and fresh and fixed tissue. Nat. Protoc. 7, 872-881 (2012).

11. Dunn, W. B. et al. Procedures for large-scale metabolic profiling of serum and plasma using gas chromatography and liquid chromatography coupled to mass spectrometry. Nat. Protoc. 6, 1060-1083 (2011).

12. Want, E. J. et al. Global metabolic profiling procedures for urine using UPLCMS. Nat. Protoc. 5, 1005-1018 (2010).

13. Rabinowitz, J. D. \& Kimball, E. Acidic acetonitrile for cellular metabolome extraction from Escherichia coli. Anal. Chem. 79, 6167-6173 (2007).

14. Bajad, S. U. et al. Separation and quantitation of water soluble cellular metabolites by hydrophilic interaction chromatography-tandem mass spectrometry. J. Chromatogr. A 1125, 76-88 (2006).

15. Lu, W. et al. Metabolomic analysis via reversed-phase ion-pairing liquid chromatography coupled to a stand alone orbitrap mass spectrometer. Anal. Chem. 82, 3212-3221 (2010).

16. DeHaven, C. D., Evans, A. M., Dai, H. \& Lawton, K. A. Organization of GC/MS and LC/MS metabolomics data into chemical libraries. J. Cheminform. 2, 9 (2010).

17. Choi, J., Grossbach, M. T. \& Antoniewicz, M. R. Measuring complete isotopomer distribution of aspartate using gas chromatography/tandem mass spectrometry. Anal. Chem. 84, 4628-4632 (2012).

18. Peterson, A. C., Balloon, A. J., Westphall, M. S. \& Coon, J. J. Development of a GC/quadrupole-orbitrap mass spectrometer, part II: new approaches for discovery metabolomics. Anal. Chem. 86, 10044-10051 (2014).

19. Lu, W. et al. Metabolite measurement: pitfalls to avoid and practices to follow. Annu. Rev. Biochem. 86, 277-304 (2017).

20. Mayers, J. R. et al. Tissue of origin dictates branched-chain amino acid metabolism in mutant Kras-driven cancers. Science 353, 1161-1165 (2016).

21. Dang, $L$. et al. Cancer-associated IDH1 mutations produce 2hydroxyglutarate. Nature 462, 739-744 (2009).

22. $\mathrm{Xu}, \mathrm{W}$. et al. Oncometabolite 2-hydroxyglutarate is a competitive inhibitor of a-ketoglutarate-dependent dioxygenases. Cancer Cell 19, 17-30 (2011).

23. Chowdhury, R. et al. The oncometabolite 2-hydroxyglutarate inhibits histone lysine demethylases. EMBO Rep. 12, 463-469 (2011).

24. Yang, Z. et al. 2-HG inhibits necroptosis by stimulating DNMT1-dependent hypermethylation of the RIP3 promoter. Cell Rep. 19, 1846-1857 (2017).

25. Losman, J-A. et al. R-2-Hydroxyglutarate is sufficient to promote leukemogenesis and its effects are reversible. Science 339, 1621-1625 (2013).
26. Folch, J., Lees, M. \& Sloane-Stanley, G. A simple method for the isolation and purification of total lipids from animal tissues. J. Biol. Chem. 226, 497-509 (1957).

27. Yetukuri, L. et al. Bioinformatics strategies for lipidomics analysis: characterization of obesity related hepatic steatosis. BMC Syst. Biol. 1, 12 (2007).

28. Yang, K. \& Han, X. Lipidomics: techniques, applications, and outcomes related to biomedical sciences. Trends Biochem. Sci. 41, 954-969 (2016).

29. Kamphorst, J. J., Fan, J., Lu, W., White, E. \& Rabinowitz, J. D. Liquid chromatography-high resolution mass spectrometry analysis of fatty acid metabolism. Anal. Chem. 83, 9114-9122 (2011).

30. Sánchez-Ávila, N., Mata-Granados, J. M., Ruiz-Jiménez, J. \& Luque de Castro, M. D. Fast, sensitive and highly discriminant gas chromatography-mass spectrometry method for profiling analysis of fatty acids in serum. J. Chromatogr. A 1216, 6864-6872 (2009).

31. Kamphorst, J. J. et al. Hypoxic and Ras-transformed cells support growth by scavenging unsaturated fatty acids from lysophospholipids. Proc. Natl Acad. Sci. USA 110, 8882-8887 (2013).

32. Svensson, R. U. et al. Inhibition of acetyl-CoA carboxylase suppresses fatty acid synthesis and tumor growth of non-small-cell lung cancer in preclinical models. Nat. Med. 22, 1108-1119 (2016).

33. DeNicola, G. M. et al. Oncogene-induced Nrf2 transcription promotes ROS detoxification and tumorigenesis. Nature 475, 106-109 (2011).

34. Zhu, P., Oe, T. \& Blair, I. A. Determination of cellular redox status by stable isotope dilution liquid chromatography/mass spectrometry analysis of glutathione and glutathione disulfide. Rapid Commun. Mass Spectrom. 22, 432-440 (2008).

35. Ortmayr, K., Schwaiger, M., Hann, S. \& Koellensperger, G. An integrated metabolomics workflow for the quantification of sulfur pathway intermediates employing thiol protection with $\mathrm{N}$-ethyl maleimide and hydrophilic interaction liquid chromatography tandem mass spectrometry. Analyst 140, 7687-7695 (2015).

36. Lu, W., Wang, L., Chen, L., Hui, S. \& Rabinowitz, J. D. Extraction and quantitation of NAD (P)(H). Antioxid. Redox Signal. 28, 167-179 (2018).

37. Locasale, J. W. Serine, glycine and one-carbon units: cancer metabolism in full circle. Nat. Rev. Cancer 13, 572-583 (2013).

38. DeNicola, G. M. et al. NRF2 regulates serine biosynthesis in non-small cell lung cancer. Nat. Genet. 47, 1475-1481 (2015).

39. Amelio, I., Cutruzzolá, F., Antonov, A., Agostini, M. \& Melino, G. Serine and glycine metabolism in cancer. Trends Biochem. Sci. 39, 191-198 (2014).

40. Labuschagne, C. F., van den Broek, N. J., Mackay, G. M., Vousden, K. H. \& Maddocks, O. D. Serine, but not glycine, supports one-carbon metabolism and proliferation of cancer cells. Cell Rep. 7, 1248-1258 (2014).

41. Chen, L., Ducker, G. S., Lu, W., Teng, X. \& Rabinowitz, J. D. An LC-MS chemical derivatization method for the measurement of five different one-carbon states of cellular tetrahydrofolate. Anal. Chem. 409, 5955-5964 (2017).

42. Ducker, G. S. et al. Reversal of cytosolic one-carbon flux compensates for loss of the mitochondrial folate pathway. Cell Metab. 23, 1140-1153 (2016).

43. Mashego, M. R. et al. MIRACLE: mass isotopomer ratio analysis of U-13Clabeled extracts. A new method for accurate quantification of changes in concentrations of intracellular metabolites. Biotechnol. Bioeng. 85, 620-628 (2004).

44. Bennett, B. D. et al. Absolute metabolite concentrations and implied enzyme active site occupancy in Escherichia coli. Nat. Chem. Biol. 5, 593-599 (2009).

45. Pavlova, N. N. \& Thompson, C. B. The emerging hallmarks of cancer metabolism. Cell Metab. 23, 27-47 (2016).

46. Bruntz, R. C., Lane, A. N., Higashi, R. M. \& Fan, T. W. Exploring cancer metabolism using stable isotope resolved metabolomics (SIRM). J. Biol. Chem. 292 11601-11609 (2017).

47. Weinberg, F. et al. Mitochondrial metabolism and ROS generation are essential for Kras-mediated tumorigenicity. Proc. Natl Acad. Sci. USA 107, 8788-8793 (2010).

48. DeBerardinis, R. J. \& Chandel, N. S. Fundamentals of cancer metabolism. Sci. Adv. 2, e1600200 (2016).

49. Jiang, L. et al. Reductive carboxylation supports redox homeostasis during anchorage-independent growth. Nature 532, 255-258 (2016).

50. Maddocks, O. D., Labuschagne, C. F., Adams, P. D. \& Vousden, K. H. Serine metabolism supports the methionine cycle and DNA/RNA methylation through de novo ATP synthesis in cancer cells. Mol. Cell 61, 210-221 (2016).

51. Yang, Y., Fan, T. W., Lane, A. N. \& Higashi, R. M. Chloroformate derivatization for tracing the fate of amino acids in cells and tissues by multiple stable isotope resolved metabolomics (mSIRM). Anal. Chim. Acta 976, 63-73 (2017). 
52. Lewis, C. A. et al. Tracing compartmentalized NADPH metabolism in the cytosol and mitochondria of mammalian cells. Mol. Cell 55, 253-263 (2014).

53. Yang, M. \& Vousden, K. H. Serine and one-carbon metabolism in cancer. Nat. Rev. Cancer 16, 650-662 (2016).

54. Lunt, S. Y. \& Vander Heiden, M. G. Aerobic glycolysis: meeting the metabolic requirements of cell proliferation. Annu. Rev. Cell Dev. Biol. 27, 441-464 (2011).

55. Hanse, E. A. et al. Cytosolic malate dehydrogenase activity helps support glycolysis in actively proliferating cells and cancer. Oncogene 36, 3915-3924 (2017).

56. Liu, L. et al. Malic enzyme tracers reveal hypoxia-induced switch in adipocyte NADPH pathway usage. Nat. Chem. Biol. 12, 345-352 (2016).

57. Hellerstein, M. K. \& Murphy, E. Stable isotope-mass spectrometric measurements of molecular fluxes in vivo: emerging applications in drug development. Curr. Opin. Mol. Ther. 6, 249-264 (2004).

58. Patra, K. C. \& Hay, N. The pentose phosphate pathway and cancer. Trends Biochem. Sci. 39, 347-354 (2014).

59. Bulusu, V. et al. Acetate recapturing by nuclear acetyl-CoA synthetase 2 prevents loss of histone acetylation during oxygen and serum limitation. Cell Rep. 18, 647-658 (2017).

60. Chen, W. W., Freinkman, E., Wang, T., Birsoy, K. \& Sabatini, D. M. Absolute quantification of matrix metabolites reveals the dynamics of mitochondrial metabolism. Cell 166, 1324-1337.e11 (2016).

61. Zhu, A., Lee, D. \& Shim, H. Metabolic positron emission tomography imaging in cancer detection and therapy response. Semin. Oncol. 38, 55-69 (2011).

62. Eschmann, S. M. et al. Prognostic impact of hypoxia imaging with $18 \mathrm{~F}$ misonidazole PET in non-small cell lung cancer and head and neck cancer before radiotherapy. J. Nucl. Med. 46, 253-260 (2005).

63. Grassi, I. et al. The clinical use of PET with 11C-acetate. Am. J. Nucl. Med. Mol. Imaging 2, 33-47 (2012).

64. Nelson, S. J. et al. Metabolic imaging of patients with prostate cancer using hyperpolarized $\left[1-{ }^{13}\right.$ C]pyruvate. Sci. Transl. Med. 5, 198ra108 (2013).

65. Albers, M. J. et al. Hyperpolarized $13 \mathrm{C}$ lactate, pyruvate, and alanine: noninvasive biomarkers for prostate cancer detection and grading. Cancer Res. 68, 8607-8615 (2008).

66. Hensley, C. T. et al. Metabolic heterogeneity in human lung tumors. Cell 164 681-694 (2016).

67. Sellers, K. et al. Pyruvate carboxylase is critical for non-small-cell lung cancer proliferation. J. Clin. Invest. 125, 687-698 (2015).

68. Fan, T. W., Lane, A. N. \& Higashi, R. M. Stable isotope resolved metabolomics studies in ex vivo tissue slices. Bio Protoc. 6, e1730 (2016).

69. Bittner, C. X. et al. High-resolution measurement of the glycolytic rate. Front. Neuroenergetics 2, 26 (2010).

70. Jamali, S. et al. Hypoxia-induced carbonic anhydrase IX facilitates lactate flux in human breast cancer cells by non-catalytic function. Sci. Rep. 5, 13605 (2015).

71. Takanaga, H., Chaudhuri, B. \& Frommer, W. B. GLUT1 and GLUT9 as major contributors to glucose influx in HepG2 cells identified by a high sensitivity intramolecular FRET glucose sensor. Biochim. Biophys. Acta 1778, 1091-1099 (2008).

72. San Martín, A. et al. Imaging mitochondrial flux in single cells with a FRET sensor for pyruvate. PLOS ONE 9, e85780 (2014).

73. San Martín, A. et al. A genetically encoded FRET lactate sensor and its use to detect the Warburg effect in single cancer cells. PLOS ONE 8, e57712 (2013).

74. Tantama, M., Martínez-François, J. R., Mongeon, R. \& Yellen, G. Imaging energy status in live cells with a fluorescent biosensor of the intracellular ATP-to-ADP ratio. Nat. Commun. 4, 2550 (2013).

75. Okumoto, S. et al. Detection of glutamate release from neurons by genetically encoded surface-displayed FRET nanosensors. Proc. Natl Acad. Sci. USA 102, 8740-8745 (2005).

76. Gu, H. et al. A novel analytical method for in vivo phosphate tracking. FEBS Lett. 580, 5885-5893 (2006).

77. Kaper, T. et al. Nanosensor detection of an immunoregulatory tryptophan influx/kynurenine efflux cycle. PLoS Biol. 5, e257 (2007).

78. Estrella, V. et al. Acidity generated by the tumor microenvironment drives local invasion. Cancer Res. 73, 1524-1535 (2013).

79. Damaghi, M., Wojtkowiak, J. \& Gillies, R. pH sensing and regulation in cancer. Front. Physiol. 4, 370 (2013)

80. Shirmanova, M. V. et al. Intracellular pH imaging in cancer cells in vitro and tumors in vivo using the new genetically encoded sensor SypHer2. Biochim. Biophys. Acta 1850, 1905-1911 (2015).
81. Matlashov, M. E. et al. Fluorescent ratiometric pH indicator SypHer2: applications in neuroscience and regenerative biology. Biochim. Biophys. Acta 1850, 2318-2328 (2015).

82. Shirmanova, M. V. et al. Chemotherapy with cisplatin: insights into intracellular $\mathrm{pH}$ and metabolic landscape of cancer cells in vitro and in vivo. Sci. Rep. 7, 8911 (2017)

83. Hung, Y. P., Albeck, J. G., Tantama, M. \& Yellen, G. Imaging cytosolic NADHNAD + redox state with a genetically encoded fluorescent biosensor. Cell Metab. 14, 545-554 (2011).

84. $\mathrm{Hu}, \mathrm{H}$. et al. Phosphoinositide 3-kinase regulates glycolysis through mobilization of aldolase from the actin cytoskeleton. Cell 164, 433-446 (2016).

85. Engelman, J. A. Targeting PI3K signalling in cancer: opportunities, challenges and limitations. Nat. Rev. Cancer 9, 550-562 (2009).

86. Cameron, W. D. et al. Apollo-NADP $(+)$ : a spectrally tunable family of genetically encoded sensors for NADP(+). Nat. Methods 13, 352-358 (2016).

87. Cheng, G. et al. Profiling and targeting of cellular bioenergetics: inhibition of pancreatic cancer cell proliferation. Br. J. Cancer 111, 85-93 (2014).

88. Ferrick, D. A., Neilson, A. \& Beeson, C. Advances in measuring cellular bioenergetics using extracellular flux. Drug Discov. Today 13, 268-274 (2008).

89. Nadanaciva, S. et al. Assessment of drug-induced mitochondrial dysfunction via altered cellular respiration and acidification measured in a 96-well platform. J. Bioenerg. Biomembr. 44, 421-437 (2012).

90. Salabei, J. K., Gibb, A. A. \& Hill, B. G. Comprehensive measurement of respiratory activity in permeabilized cells using extracellular flux analysis. Nat. Protoc. 9, 421-438 (2014).

91. Miller, A. et al. Exploring metabolic configurations of single cells within complex tissue microenvironments. Cell Metab. 26, 788-800 (2017).

92. Kottakis, F. et al. LKB1 loss links serine metabolism to DNA methylation and tumorigenesis. Nature 539, 390-395 (2016).

93. Kim, J. et al. CPS1 maintains pyrimidine pools and DNA synthesis in KRAS/ LKB1-mutant lung cancer cells. Nature 546, 168-172 (2017).

94. Navin, N. et al. Tumour evolution inferred by single-cell sequencing. Nature 472, 90-94 (2011).

95. Shapiro, E., Biezuner, T. \& Linnarsson, S. Single-cell sequencing-based technologies will revolutionize whole-organism science. Nat. Rev. Genet. 14, 618-630 (2013).

96. Wang, Y. et al. Clonal evolution in breast cancer revealed by single nucleus genome sequencing. Nature 512, 155-160 (2014).

97. $\mathrm{Xu}, \mathrm{X}$. et al. Single-cell exome sequencing reveals single-nucleotide mutation characteristics of a kidney tumor. Cell 148, 886-895 (2012).

98. Heath, J. R., Ribas, A. \& Mischel, P. S. Single-cell analysis tools for drug discovery and development. Nat. Rev. Drug Discov. 15, 204-216 (2015).

99. Irish, J. M., Kotecha, N. \& Nolan, G. P. Mapping normal and cancer cell signalling networks: towards single-cell proteomics. Nat. Rev. Cancer 6, 146-155 (2006).

100. Lombard-Banek, C., Moody, S. A. \& Nemes, P. Single-cell mass spectrometry for discovery proteomics: quantifying translational cell heterogeneity in the 16Cell Frog (Xenopus) embryo. Angew. Chem. Int. Ed. Engl. 55, 2454-2458 (2016).

101. Altelaar, A. F. \& Heck, A. J. Trends in ultrasensitive proteomics. Curr. Opin. Chem. Biol. 16, 206-213 (2012).

102. Zenobi, R. Single-cell metabolomics: analytical and biological perspectives. Science 342, 1243259 (2013).

103. Amantonico, A., Urban, P. L. \& Zenobi, R. Analytical techniques for single-cell metabolomics: state of the art and trends. Anal. Bioanal. Chem. 398, 2493-2504 (2010).

104. Do, T. D., Comi, T. J., Dunham, S. J. B., Rubakhin, S. S. \& Sweedler, J. V. Single cell profiling using ionic liquid matrix-enhanced secondary ion mass spectrometry for neuronal cell type differentiation. Anal. Chem. 89, 3078-3086 (2017).

105. Feist, P. \& Hummon, A. B. Proteomic challenges: sample preparation techniques for microgram-quantity protein analysis from biological samples. Int J. Mol. Sci. 16, 3537-3563 (2015).

106. Svatoš, A. Single-cell metabolomics comes of age: new developments in mass spectrometry profiling and imaging. Anal. Chem. 83, 5037-5044 (2011).

107. Wang, D. \& Bodovitz, S. Single cell analysis: the new frontier in 'omics'. Trends Biotechnol. 28, 281-290 (2010).

108. Shalem, O., Sanjana, N. E. \& Zhang, F. High-throughput functional genomics using CRISPR-Cas9. Nat. Rev. Genet. 16, 299 (2015).

109. Shalem, O. et al. Genome-scale CRISPR-Cas9 knockout screening in human cells. Science 343, 84-87 (2014). 
110. Wang, T. et al. Identification and characterization of essential genes in the human genome. Science 350, 1096-1101 (2015).

111. Birsoy, K. et al. An essential role of the mitochondrial electron transport chain in cell proliferation is to enable aspartate synthesis. Cell 162, 540-551 (2015).

112. Kerr, E. M., Gaude, E., Turrell, F. K., Frezza, C. \& Martins, C. P. Mutant Kras copy number defines metabolic reprogramming and therapeutic susceptibilities. Nature 531, 110-113 (2016).

113. Sanchez-Rivera, F. J. et al. Rapid modelling of cooperating genetic events in cancer through somatic genome editing. Nature 516, 428-431 (2014).

114. Mazur, P. K. et al. Combined inhibition of BET family proteins and histone deacetylases as a potential epigenetics-based therapy for pancreatic ductal adenocarcinoma. Nat. Med. 21, 1163-1171 (2015).

115. Romero, R. et al. Keap1 loss promotes Kras-driven lung cancer and results in dependence on glutaminolysis. Nat. Med. 23, 1362-1368 (2017).

116. Zhang, $H$. et al. Distinct metabolic states can support self-renewal and lipogenesis in human pluripotent stem cells under different culture conditions. Cell Rep. 16, 1536-1547 (2016).
117. Cantor, J. R. et al. Physiologic medium rewires cellular metabolism and reveals uric acid as an endogenous inhibitor of UMP synthase. Cell 169 258-272.e17 (2017).

118. Birsoy, K. et al. Metabolic determinants of cancer cell sensitivity to glucose limitation and biguanides. Nature 508, 108-112 (2014).

119. Lancaster, M. A. \& Knoblich, J. A. Organogenesis in a dish: modeling development and disease using organoid technologies. Science 345, 1247125 (2014).

120. Gao, D. et al. Organoid cultures derived from patients with advanced prostate cancer. Cell 159, 176-187 (2014).

121. Boj, S. F. et al. Organoid models of human and mouse ductal pancreatic. Cancer Cell 160, 324-338 (2015).

122. van de Wetering, M. et al. Prospective derivation of a living organoid biobank of colorectal cancer patients. Cell 161, 933-945 (2015).

123. Walsh, A. J. et al. Quantitative optical imaging of primary tumor organoid metabolism predicts drug response in breast cancer. Cancer Res. 74, 5184-5194 (2014). 